\title{
Connecting the macro- and microstrain responses in technical porous ceramics: modeling and experimental validations
}

\author{
Giovanni Bruno • Alexander M. Efremov • \\ Andreyi N. Levandovskyi $\cdot$ Bjorn Clausen
}

Received: 22 January 2010/Accepted: 4 September 2010/Published online: 28 September 2010

(C) Springer Science+Business Media, LLC 2010

\begin{abstract}
The relation between the macroscopic and the microscopic (lattice) strain response to external uniaxial stress has been investigated for porous ceramics. Analytical and finite element modeling (FEM) have been performed and neutron diffraction data on porous sintered alumina and extruded honeycomb $\mathrm{SiC}$ have been used to validate the theoretical approach. By FEM simulations, it is shown that in spite of the complex pore microstructure, shear stresses are small during uniaxial compression. Analytical modeling shows that while the average microscopic stress depends on the applied macroscopic stress only through the porosity $p$, the average microscopic strain depends on the macroscopic stress through the pore morphology factor $m$, as well. Novel relationships are proposed to describe this dependence. Analytical calculations and numerical modeling perfectly agree with each other, and both show good consistency with experiments. As predicted, it has been observed that the microscopic (diffraction) Young's modulus does not depend on the pore morphology factor, and follows the rule-of-mixtures, while the microscopic Poisson's ratio does not even depend on porosity, but is equal to the value for the dense material property. A practical implication of these findings is that it is not possible to
\end{abstract}

\footnotetext{
G. Bruno $(\bowtie)$

Corning SAS, CETC, Materials, 7 bis Av.de Valvins,

77210 Avon, France

e-mail: brunog@corning.com
}

A. M. Efremov - A. N. Levandovskyi

Corning Inc., CSC 26 liter A, Shatelena Str.,

194018 St Petersburg, Russia

B. Clausen

Lujan Center, LANSCE, LANL, Los Alamos 87545, USA attach a pore morphology factor to a material, unless the processing conditions are tailored to vary $p$ without varying $m$. In fact, the different values of $m$ found for the different porosities explain why many models can be used to rationalize the experimental data. With the proposed method, the factor $m$ can be independently evaluated by the use of macro- and micro-elastic properties of the porous body. Analogously, the macroscopic elastic properties of the dense material can be obtained by macroscopic and microscopic values measured on the correspondent porous material.

\section{Introduction}

A very large amount of data is available in the literature about the macrostrain response to applied stress of porous materials (see [1-6] for some examples). In particular, the works of Knudsen [2], Dean and Lopez [5], and Munro [6] on alumina outline that several models (exponential, polynomial, and even linear) can be fitted to the experimental data, especially if porosities $p<0.5$ are considered. In his book, Green reports that linear Young's modulus reduction as a function of porosity can be interpreted in the frame of a percolation theory [7]. Further, Wang [8] and Sudduth [9] discuss the limits of validity of different hypotheses. Ramakrishnan and Arunachalam [10] point out that one should refrain from using a simplistic composite model with the void having zero mechanical properties. A useful approach is also given by Roberts and Garboczi [11]: these authors derive analytical formulae (power laws) for the cases of spherical and ellipsoidal pores, as well as for overlapping matrix spheroid particles. They use different percolation levels (i.e., porosity values at which the Young's modulus drops to zero) in the three cases. 
Thus, whereas all literature models agree that the macroscopic elastic modulus of a porous material is proportional to the elastic properties of the solid domain, they disagree about the dependence on porosity. Indeed, the pore structure (shape, size distribution or connectivity) significantly influences mechanical properties as well as porosity. This implies that a universal dependence of mechanical properties on porosity must include at least two parameters: one accounting for porosity (scalar) and one for pore morphology (which might be a tensor [12]).

To our knowledge, only a few papers tackle the question of the microscopic (lattice) response [13, 14] of porous media to external loads. It has already been found experimentally, using neutron diffraction (ND), that the microscopic stress-strain behavior of natural (porous) rocks is very different from the macroscopic. This behavior is commonly explained by the influence of porosity, but little insight has been given to clarify the mechanisms. Even further, very little modeling work is available on the lattice strain behavior [15], so that data are not yet rationalized in a widely accepted coherent frame.

The relationship linking an applied macroscopic stress to the macroscopic strain and to the lattice response is, however, of great importance in order to: (a) understand and quantify the role of materials properties in the specimen or component response, and (b) infer the macroscopic behavior from microscopic data (or vice versa), as in the case of a crack initiation or propagation criterion. Commonly, the average microscopic and macroscopic moduli are assumed equal for a dense material. However, they do differentiate in porous ceramic materials and it is not obvious that the properties of solid skeleton are the same as the properties of the analogous dense material. In fact, while for dense materials analytical models exist to calculate the polycrystalline average microscopic (crystallographic plane specific) elastic properties [16, 17], this is not the case when pores represent one of the phases (see e.g., [18], where a mean field approach is used, but not crystal plane-specific).

Recently, new microstructural information has allowed further progress. Indeed, inputs to stress-strain modeling have been expanded to microscopic data such as the threedimensional (3D) pore structure, retrievable for example by means of synchrotron or table X-ray tomography [19]. In this framework, microcrack behavior under applied stress has also been used, as measured by SEM and TEM [20]. Other powerful instruments, such as focused ion beam (FIB) chambers are being widely used to acquire microstructural information. These techniques, however, do not yield any quantitative information about the material's mechanical properties.

In this work, the relationship between macroscopic and microscopic properties has been analytically modeled using stress balance considerations. We started from a simple empirical relationship (introduced by Gibson and Ashby [21]) between the Young's modulus $E_{\mathrm{p}}$ of a porous body and its dense material properties $\left(E_{\mathrm{d}}\right)$, assumed uniform and isotropic. Indeed, a one-parameter model cannot cope with the anisotropy of the pore structure, since porosity is a scalar quantity. At least one more model parameter is required for an adequate description of the influence of the pore structure on elastic properties. We chose this second parameter to be the pore morphology factor $m$. While studying porous solids with open and closed cells, Gibson and Ashby used the parameter $m$ to characterize the cells and derived $m=2$ and $m=3$, respectively, for the two cases above.

The approach has proved promising and deserving extension to other porous ceramics. Therefore, we have made use of the parameter $m$ in a broader sense, to define open (continuous) porosity. This parameter would be analogous to the pore shape for closed porosity. A relationship has been derived, linking the average microscopic lattice strain (microstrain) measured by diffraction methods, to the macroscopic strain (macrostrain) measured by extensometry or strain gauges. Some direct measurements of the microscopic and macroscopic strain response under external applied stress in porous honeycomb silicon carbide and porous compact alumina have been carried out as examples. We will show that the combined experiment plus modeling approach and the addition of microscopic (diffraction) data to macroscopic tests, both cast new light on the micromechanics of porous ceramics. We will also see that the different values of $m$ found for the different porosities explain why many models could and can be used to rationalize the experimental data.

\section{Model description and predictions}

Analytical relations for micro- to macrostrain conversion

A model for the conversion of macro- to micro-elastic properties in bi-continuous porous structures with elastically isotropic solid properties can be derived from stress balance principles (see Fig. 1). We consider a unit volume of a linear elastic porous medium and assume that the dependence of the Young's modulus of the porous material, $E_{\mathrm{p}}$, on the porosity, $p$, is given by

$E_{\mathrm{p}}=E_{\mathrm{d}} \cdot(1-p)^{m}$

where $m$ denotes the pore morphology factor defined in [21], and $E_{\mathrm{d}}$ denotes the Young's modulus of the solid domain in the material (commonly assumed to coincide with the measured dense material Young's modulus). We chose this model as it is a simple way to explicitly include 


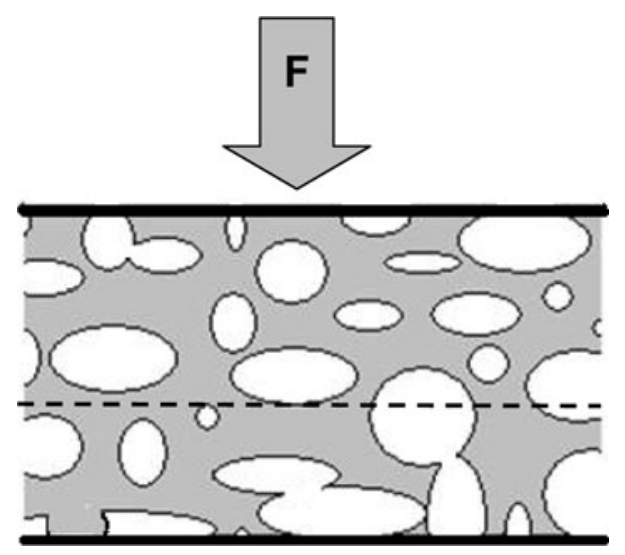

Fig. 1 A sketch of the macro-microstress balance of a porous body under external uniaxial stress. $F$ indicates the external force, which distributes over the solid domain of any transverse cut (dashed line), proportionally to the porosity

the influence of porosity and pore morphology. We will show that our results ( $m=2$ for spherical pores) reproduce previous work for porous glass and ceramic [11, 21-23].

The macroscopic strain $\varepsilon_{\text {Macro }}$ due to an applied macroscopic uniaxial stress $\sigma_{\text {Macro }}$ follows tensorial Hooke's law

$\sigma_{\text {Macro }}=E_{\mathrm{p}} \cdot \varepsilon_{\text {Macro }}$

Analogously, the local microstrain $\varepsilon_{\text {micro }}$ due to the local microstress $\sigma_{\text {micro }}$ in each point of the dense skeleton is given by tensorial equation

$\sigma_{\text {micro }}=E_{\mathrm{d}} \cdot \varepsilon_{\text {micro }}$

We now apply Gauss-Ostrogradskiy's divergence theorem to the stress field in our volume (Fig. 1): the stress integral over any cross-section perpendicular to the load direction is equal to the externally applied force [24]. The latter is simply equal to the macroscopic stress times the whole cross-section area, including pores. This can be expressed in terms of macrostress and average microstress by

$\left\langle\sigma_{\text {micro }}\right\rangle=\frac{\sigma_{\text {Macro }}}{1-p}$

(Here and below angle brackets denote averaging over the solid domain and the component normal to the crosssection has been taken.)

In other words, Eq. 4 implies that the average microstress measured by ND along the load direction equals the applied stress normalized by the fraction of the solid domain.

From Eqs. 1-4, the micro- to macrostress scalar relationships for strain and stress are derived as

$\frac{\left\langle\sigma_{\text {micro }}\right\rangle}{\sigma_{\text {Macro }}}=(1-p)^{-1}$ $\frac{\left\langle\varepsilon_{\text {micro }}\right\rangle}{\varepsilon_{\text {Macro }}}=\frac{1}{E_{\mathrm{d}}} \frac{\left\langle\sigma_{\text {micro }}\right\rangle}{\varepsilon_{\text {Macro }}}=\frac{E_{\mathrm{p}}}{E_{\mathrm{d}}} \frac{\left\langle\sigma_{\text {micro }}\right\rangle}{\sigma_{\text {Macro }}}=(1-p)^{m-1}$

where $E_{\mathrm{d}}$ is a scalar elastic constant of the solid domain in the load direction, defined by $E_{\mathrm{d}}=\left\langle\sigma_{\text {micro }}\right\rangle /\left\langle\varepsilon_{\text {micro }}\right\rangle . E_{\mathrm{d}}$ has the meaning of an average microscopic Young's modulus, which is here assumed to be the same as the macroscopic modulus for the dense material. Both Eqs. 5 and 6 are general and can be used independently of the choice of the elastic model for porous media, except for the last equality in Eq. 6.

While Eq. 5 has been mentioned in the work of Kachanov et al. [12], to the best of our knowledge, Eq. 6 has been derived in this context for the first time. They imply that the average microstress is higher than the macrostress in porous media and does not depend on pore morphology. The average microstrain, e.g., measured by ND, is lower than the macroscopic value and does depend on pore morphology. Therefore, the model allows the estimation of microscale strain and stress, responsible for crack initiation and, ultimately, for failure, as a function of the applied macroscopic load, as long as $m, E_{\mathrm{d}}$, and $p$ are known. Conversely, if the properties of the porous material are available (i.e., $p, \varepsilon_{\text {Macro }}$, $\sigma_{\text {Macro }}$, and $\left\langle\varepsilon_{\text {micro }}\right\rangle$ are measured) the dense material properties and pore morphology $(m)$ can be evaluated.

Finite element modeling calculation of microstrain

In order to validate the analytical formulae (Eqs. 5 and 6) of conversion between micro- and macrostress and strain, we ran a comparison with FEM calculations, thereby using a completely independent approach.

We considered several artificial structures of various porosities and used two different pore morphologies: overlapping spherical pores (OSP) and overlapping solid spheres (OSS). This is very similar to what Roberts and Garboczi [11] or Rossi [25] did in their pioneering works. The aim of this approach was not to simulate real structures, but rather to set boundaries where the behavior of bi-continuous porous materials may fall. It is to be noted that porous microcracked materials could possibly fall in these boundaries. Similarly, the input material properties do not necessarily correspond to a real case, although we used typical Young's moduli and Poisson's ratios close to those of dense alumina.

The finite element model of the pore structure consisted of cubic volume pixels (voxels) of the same size and with isotropic mechanical properties (Fig. 2a).

The calculations were done with the program ANSYS, using the inputs shown in Table 1 (Young's modulus and Poisson's ratio of the elements). The FEM statement is depicted in Fig. $2 b$. 
Fig. 2 a OSP and OSS model structures and cubic (voxel) mesh. b FEM task statement and derivation of macroscopic values
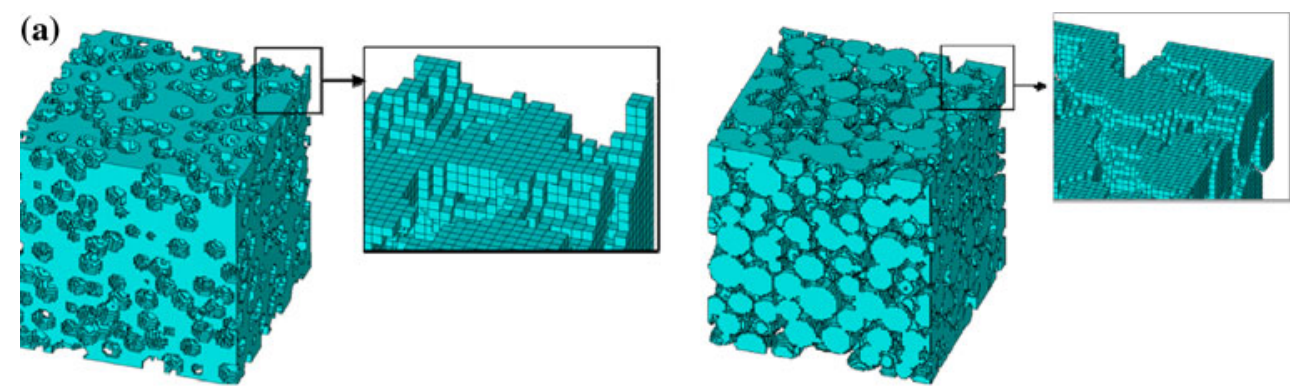

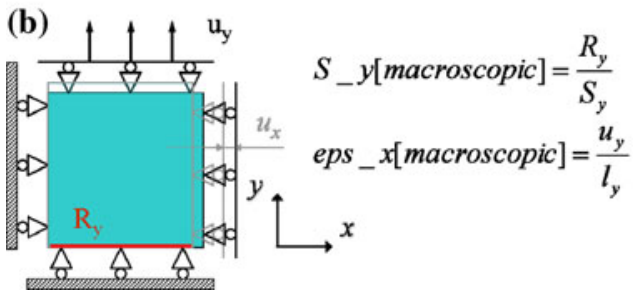

Table 1 Input data for FEM simulations

\begin{tabular}{lll}
\hline$d$ & Element size, micron & 1 \\
$E_{\mathrm{d}}$ & Element Young's Modulus, GPa & 395 \\
$v_{\mathrm{d}}$ & Element Poisson ratio & 0.23 \\
$n$ & Structure cubic sample size in elements & $100^{3}$ \\
eps_y & Macroscopic uniaxial strain applied $(y), \mathrm{ppm}$ & 1000 \\
\hline
\end{tabular}

Stress corresponding to a uniaxial strain of $1000 \mu \varepsilon$ (or ppm) was applied along the $y$-axis of Fig. $2 b$ and the (linear) response of the body along the principal axes $x, y$, $z$ was calculated. The microscopic properties were calculated for each voxel and then averaged over all elements across the structure.

The results of the FEM calculations are given in Table 2. While we found a uniaxial macroscopic stress state consistent with the analytical conditions, we also obtained some non-zero microscopic shear stresses and principal strain components (see Table 2). From the macroand microstrains and stresses, we calculated the ratios micro/macro, as well as the macroscopic and microscopic Poisson's ratios and the apparent diffraction modulus, i.e., the slope of the curve $\sigma_{\text {Macro }}$ versus $\left\langle\varepsilon_{\text {micro }}\right\rangle$.

Interestingly, the microscopic Poisson's ratio $\left\langle\varepsilon_{x}\right\rangle /$ $\left\langle\varepsilon_{y}\right\rangle=0.23$ turns out to be invariant on porosity and pore morphology, and corresponds exactly to the input value $v_{\mathrm{d}}=0.23$.

The relative difference of the Poisson's ratio and other quantities, as calculated with the analytical and with the numerical approaches, is astoundingly small, of the order of a few ppm: e.g., $\Delta v / v \sim 10^{-6}$. This confirms the validity of the analytical model set forth above.

Figure 3 compares the analytical model, Eq. 6, with FEM results by plotting the average micro- to macrostrain ratio as a function of porosity. One can see the FEM simulation results agree perfectly with the analytical model if $m=2$ for OSP and $m \sim 4$ for OSS. These findings are also in perfect agreement with the values for commercial ceramics proposed by Pabst and Gregorová [4] and with the work of Roberts and Garboczi [11] on structures with non-spherical pores. In Fig. 3, the experimental value obtained for $\mathrm{SiC}$ (described below) is also reported. We will see that it fits very well in the frame drawn here.

\section{Experimental validation}

\section{Samples}

In order to validate the model proposed, we chose nonmicrocracked $\mathrm{SiC}$ and $\mathrm{Al}_{2} \mathrm{O}_{3}$ materials (the first also reported in [26]), as they display a basically linear elastic response and low elastic (lattice) anisotropy. We used commercially available $\mathrm{SiC}$ honeycomb extruded filter specimens and powder sintered alumina samples. For the latter, the porosity variation was obtained simply by varying the sintering time and pressure, using the same alumina raw material ( $\sim 1 \mu \mathrm{m}$ particle size $)$. An example of the microstructure, a backscattered electron micrograph of the SiC sample is shown in Fig. 4. Interestingly enough, but expectedly, the grain and pore shapes show a microstructure between the OSP and OSS.

The $\mathrm{SiC}$ samples were cut from a $152-\mathrm{mm}$ honeycomb filter as rectangular prisms with dimension of about $12 \times 25 \times 50 \mathrm{~mm}$ (compression test specimens). The cell structure dimensions of the filter (see also Fig. 5a), as well as the relevant geometrical and physical properties, are provided in Table 3. 
Table 2 Results of FEM simulations and derived micro/macro relationships as a function of porosity and pore shape

\begin{tabular}{|c|c|c|c|c|c|c|c|c|c|}
\hline & \multirow[t]{2}{*}{ ID } & \multirow{2}{*}{$\begin{array}{l}\text { Parameterl|structure } \\
\text { Porosity }(p)\end{array}$} & \multicolumn{4}{|c|}{ Overlapping pores } & \multicolumn{3}{|c|}{ Overlapping spheres } \\
\hline & & & 0.30 & 0.40 & 0.50 & 0.60 & 0.63 & 0.51 & 0.40 \\
\hline \multirow[t]{12}{*}{ Average Microscopic } & SX & Tang. stress $(x), \mathrm{MPa}$ & 0 & 0 & 0 & 0 & 0 & 0 & 0 \\
\hline & SY & Axial stress (y), MPa & 273 & 230 & 193 & 152 & 17 & 46 & 106 \\
\hline & $\mathrm{SZ}$ & Tang. stress (z), MPa & 0 & 0 & 0 & 0 & 0 & 0 & 0 \\
\hline & SXY & Shear stress $(x y), \mathrm{MPa}$ & 2 & 2 & -1 & 0 & 0 & 0 & 0 \\
\hline & SYZ & Shear stress $(y z), \mathrm{MPa}$ & -1 & -1 & 0 & -1 & 0 & 0 & 0 \\
\hline & SXZ & Shear stress $(x z), \mathrm{MPa}$ & 0 & 0 & 0 & 0 & 0 & 0 & 0 \\
\hline & EPSX & Tang. strain $(x)$, ppm & -159 & -134 & -112 & -89 & -10 & -27 & -62 \\
\hline & EPSY & Axial strain $(y)$, ppm & 690 & 583 & 488 & 386 & 43 & 116 & 269 \\
\hline & EPSZ & Tang. strain $(z), \mathrm{ppm}$ & -159 & -134 & -112 & -89 & -10 & -27 & -62 \\
\hline & EPSXY & Shear strain $(x y)$, ppm & 10 & 11 & -5 & 0 & -3 & -2 & 0 \\
\hline & EPSYZ & Shear strain $(y z)$, ppm & -5 & -6 & -1 & -5 & -1 & 1 & -3 \\
\hline & EPSXZ & Shear strain $(x z)$, ppm & 0 & -2 & -1 & -1 & -1 & 2 & -2 \\
\hline \multirow[t]{6}{*}{ Macroscopic } & S_x & Tang. stress $(x), \mathrm{MPa}$ & 0 & 0 & 0 & 0 & 0 & 0 & 0 \\
\hline & S_y & Axial stress $(y), \mathrm{MPa}$ & 191 & 138 & 96 & 61 & 6 & 23 & 64 \\
\hline & S_z & Tang. stress $(z), \mathrm{MPa}$ & 0 & 0 & 0 & 0 & 0 & 0 & 0 \\
\hline & eps_x & Tang. strain $(x)$, ppm & -219 & -213 & -220 & -215 & -161 & -154 & -174 \\
\hline & eps_y & Axial strain $(y), \mathrm{ppm}$ & 1000 & 1000 & 1000 & 1000 & 1000 & 1000 & 1000 \\
\hline & eps_z & Tang. strain $(z)$, ppm & -218 & -221 & -219 & -224 & -170 & -164 & -173 \\
\hline \multirow[t]{7}{*}{ Derived values } & & Axial Young's modulus $\left(E_{\mathrm{p}}\right), \mathrm{GPa}$ & 191 & 138 & 96 & 61 & 6 & 23 & 64 \\
\hline & & Pore morphology factor $(m)$ & 2.04 & 2.05 & 2.03 & 2.04 & 4.15 & 4.05 & 3.57 \\
\hline & & Poisson ratio $\left(v_{\mathrm{p}}\right)$ & 0.22 & 0.22 & 0.22 & 0.22 & 0.17 & 0.16 & 0.17 \\
\hline & & $\left\langle\sigma_{\text {micro }}\right\rangle / \sigma_{\text {Macro }}$ & 1.43 & 1.67 & 2.00 & 2.50 & 2.71 & 2.03 & 1.67 \\
\hline & & $\left\langle\varepsilon_{\text {micro }}\right\rangle / \varepsilon_{\text {Macro }}$ & 0.69 & 0.58 & 0.49 & 0.39 & 0.04 & 0.12 & 0.27 \\
\hline & & Diffraction modulus, GPa & 276 & 237 & 197 & 158 & 146 & 195 & 237 \\
\hline & & $\left\langle\varepsilon_{\text {micro }}\right\rangle x, z /\left\langle\varepsilon_{\text {micro }}\right\rangle y$ & 0.23 & 0.23 & 0.23 & 0.23 & 0.23 & 0.23 & 0.23 \\
\hline
\end{tabular}

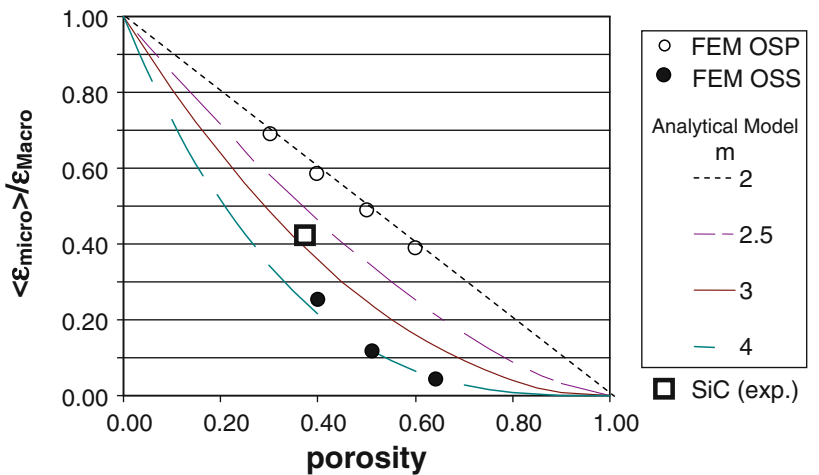

Fig. 3 The comparison of our analytical model, Eq. 6, and FEM simulations as a function of porosity and pore morphology: overlapping spherical pores (OSP), overlapping solid spheres (OSS)

Various alumina cylinders (diameter about $12 \mathrm{~mm}$ and length about $19 \mathrm{~mm}$ ) with different porosities were produced. The sample dimensions as well as the relevant geometrical and physical properties are given in Table 4 . The modulus of rupture (MOR) was measured by uniaxial compression tests on sister samples and using the same

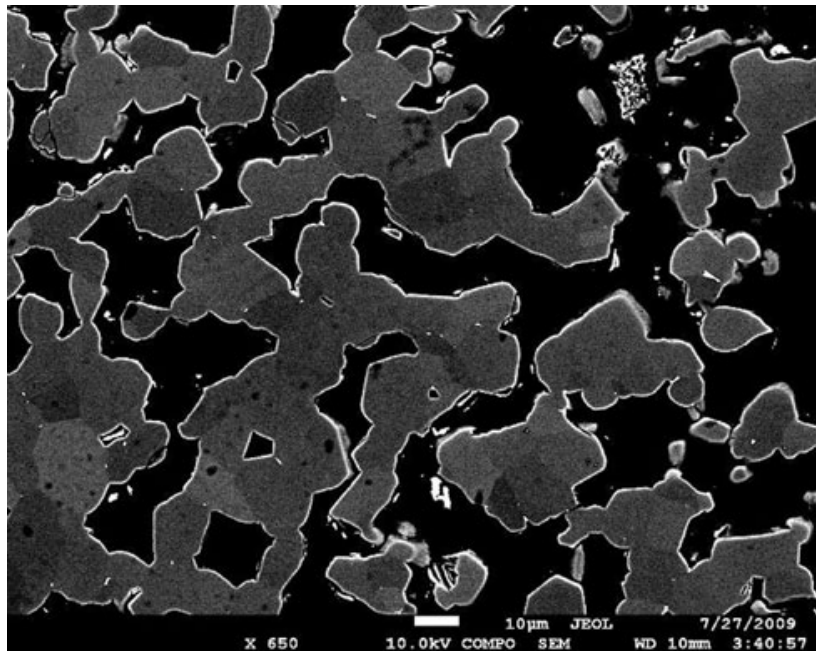

Fig. 4 SEM picture of the microstructure of the $\mathrm{SiC}$ material investigated. The contrast of the grains in the backscattered electron imaging is due to the different orientation

machine used for in situ ND. The porosity $p$ and the pore size $d_{50}$ were measured by Mercury Intrusion on an AutoporeSizer 9520, Micromeritics. 

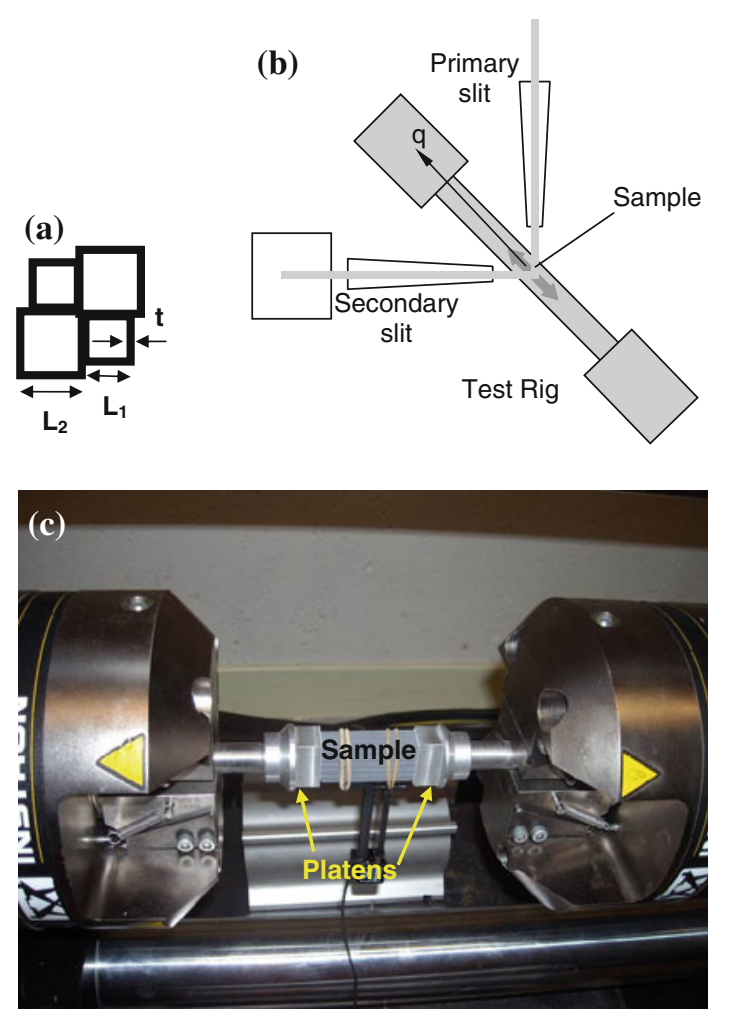

Fig. 5 a Sketch of the asymmetric cell geometry of the SiC sample, with definition of the dimensions of Table 3. b Sketch of the test rig set-up and the sample mount on the SALSA diffractometer. The $q$ vector indicates the direction of the strain under investigation. c Photo of the compression sample mount on SALSA at the ILL (specimen cut from a honeycomb commercial filter)

\section{ND in situ experiments}

In order to probe the bulk lattice response and avoid unwanted surface effects, we used ND. Diffraction methods easily allow the determination of the crystal strain response; several textbooks are available on the subject $[27,28]$. In the case of ND, the high penetration of the radiation allows probing the bulk of the sample. As a further advantage over synchrotron radiation, by means of tailored gauge volumes (such as $5 \times 10 \times 5 \mathrm{~mm}$ ), the average response over a sufficiently large statistical grain ensemble can be measured. In the case of $\mathrm{SiC}$, the large gauge volume also minimizes the effect of the voids of the cellular geometry on the diffraction peak shift [29]. References [27, 28] more thoroughly describe how diffraction peak shift is used to calculate residual and applied
Table 4 Geometrical and physical parameters of the alumina samples investigated

\begin{tabular}{lllrlll}
\hline Sample & $\varnothing(\mathrm{mm})$ & $H(\mathrm{~mm})$ & $\begin{array}{l}A_{\text {eff }} \\
\left(\mathrm{mm}^{2}\right)\end{array}$ & $\begin{array}{l}\text { MOR } \\
(\mathrm{MPa})\end{array}$ & $p$ & $\begin{array}{l}d_{50} \\
(\mu \mathrm{m})\end{array}$ \\
\hline Bulk & 12.6 & 19.4 & 125.5 & 1050 & 0 & - \\
$17 \%$ & 12.2 & 18.5 & 116.3 & - & 0.17 & - \\
$33 \%$ & 10.5 & 18.5 & 87.5 & 446 & 0.33 & 7.3 \\
$43 \%$ & 11.3 & 19.3 & 100.5 & 116 & 0.44 & 8.0 \\
\hline
\end{tabular}

They were cylinders of height $H$ and diameter $\varnothing$. They are labeled after their porosity

stress. In this context we remind the reader that at a steadystate (reactor) source the microscopic strain for a particular set of lattice planes $\{h k l\} d_{h k l}$ is calculated by means of

$\varepsilon_{h k l}=\left(\frac{d_{h k l}-d_{0, h k l}}{d_{0, h k l}}\right)$

whereby $d$ and $d_{0}$ are the strained and reference $\{h k l\}$ interplanar distances, respectively. They are calculated from Bragg's law $\lambda=2 d \sin \theta$, where $\lambda$ is the neutron wavelength and $2 \theta$ the Bragg (diffraction) angle. The reference $d_{0}$ was taken as the interplanar spacing measured at zero applied load.

Diffraction experiments on the $\mathrm{SiC}$ samples were carried out on the instruments Strain Analyzer for Large and Small Engineering Applications (SALSA) at the ILL, Institut Laue-Langevin, Grenoble, France [30]. The stress rig was mounted with the scattering vector $q$ oriented along the axial and the radial sample direction. The axial mount is sketched in Fig. 5b, the radial mount implies a rotation of the rig by $90^{\circ}$. For the compressive tests we took 12 points upon loading and 6 upon unloading, using a load rate of $\sim 10 \mathrm{MPa} / \mathrm{min}$. Diffraction spectra were acquired at fixed load for about $5 \mathrm{~min}$. In order to measure the microstrain in the transverse direction, a sister sample had to be measured. In spite of the use of a position-sensitive detector with about $4^{\circ}$ width in $2 \theta$, only the hexagonal SiC-116 peak could be used. This proved to be at $2 \theta \sim 72^{\circ}$ for the wavelength used $(\lambda=0.1549 \mathrm{~nm})$. The diffraction peaks were fitted with simple Gaussian functions and the errors on the fit parameters (peak position, integral width, and area) were taken as the statistical errors. Figure 5 also shows a photo (c) of the setup used with the rig mount on SALSA's hexapod.

Table 3 Geometrical and physical parameters of the SiC sample, a cellular bar of cross-section $b \times w$, cell size $L_{1}, L_{2}$, and wall thickness $t$ (see Fig. 5a)

\begin{tabular}{|c|c|c|c|c|c|c|c|c|}
\hline \multicolumn{2}{|l|}{ Sample } & \multicolumn{3}{|l|}{ Cell } & \multirow[t]{2}{*}{$A_{\text {eff }}\left(\mathrm{mm}^{2}\right)$} & \multirow[t]{2}{*}{ MOR (MPa) } & \multirow[t]{2}{*}{$p$} & \multirow[t]{2}{*}{$d_{50}(\mu \mathrm{m})$} \\
\hline$b(\mathrm{~mm})$ & $w(\mathrm{~mm})$ & $t(\mathrm{~mm})$ & $L_{1}(\mathrm{~mm})$ & $L_{2}(\mathrm{~mm})$ & & & & \\
\hline 15.4 & 23.2 & 0.40 & 1.60 & 2.35 & 130.5 & 268 & 0.38 & 6.7 \\
\hline
\end{tabular}


In the case of pulsed neutron sources, the least-square refinement of the diffraction pattern is converted into a certain lattice parameter $a$ through Bragg's law, written for a constant diffraction angle $\left(2 \theta=90^{\circ}\right)$ but variable flight path $L$ and neutron travel time $t$ (or wavelength $\lambda$ ): $\sqrt{2} \cdot a=\frac{h \cdot t}{m_{\mathrm{n}} \cdot L}$, where $m_{\mathrm{n}}$ is the neutron mass and $h$ Planck's constant. Usually, the reference value (with index 0 ) is taken as the zero-load state and thus the average lattice strain is calculated as:

$\varepsilon_{a}=\frac{a-a_{0}}{a_{0}}$

whereby $a$ stands for any of the lattice unit cell parameters. In our case, a Rietveld refinement has been employed [31] and the lattice parameters $a$ and $c$ of rhombohedral alumina have been extracted, using the program GSAS [32].

For the alumina samples, ND measurements were conducted using the Spectrometer for Material Research at Temperature and Stress (SMARTS) instrument at Los Alamos National Laboratory (LANL) [33]. SMARTS utilizes the pulsed neutron source at Los Alamos Neutron Science Center (LANSCE) for simultaneous time-of-flight measurements of full diffraction patterns (with wavelength range between 0.04 and $0.38 \mathrm{~nm}$ ) in two detector banks oriented at $\pm 90^{\circ}$ to the incident beam. The samples were always oriented such that the loading direction was at $45^{\circ}$ to the incident beam, and thus the two detector banks allowed measuring the axial (longitudinal) and transverse direction at once. This is schematically shown in Fig. 6a: the scattering vectors $q_{\mathrm{L}}$ and $q_{\mathrm{T}}$ are aligned along the longitudinal and transverse sample directions. The gauge volume was defined by the sample dimensions and by $5 \times 10 \mathrm{~mm}$ incident (flat) slits, as no collimation was used on the diffracted beam (see sketch in Fig. 6a).

A hydraulic Instron test rig was used (see Fig. 6b), with two load cells: $10 \mathrm{kN}$ for the $43 \%$ porosity $\mathrm{Al}_{2} \mathrm{O}_{3}$ and $250 \mathrm{kN}$ for the $0 \%, 17$ and $33 \%$ porosity $\mathrm{Al}_{2} \mathrm{O}_{3}$ samples. Steel compression platens with cylindrical shape were especially built and a paper foil was always interposed between the platens and the sample ends. A high resolution Instron extensometer was used to measure the macroscopic strain (Fig. 6b), attached to the samples using rubber bands.

\section{Experimental results}

The macro- and microstrains (Si-116) recorded on SALSA are plotted against the applied stress in Fig. 7. Several features can be remarked:

- The microstrain behavior is almost perfectly linear within the data scatter. More data scatter occurs in the
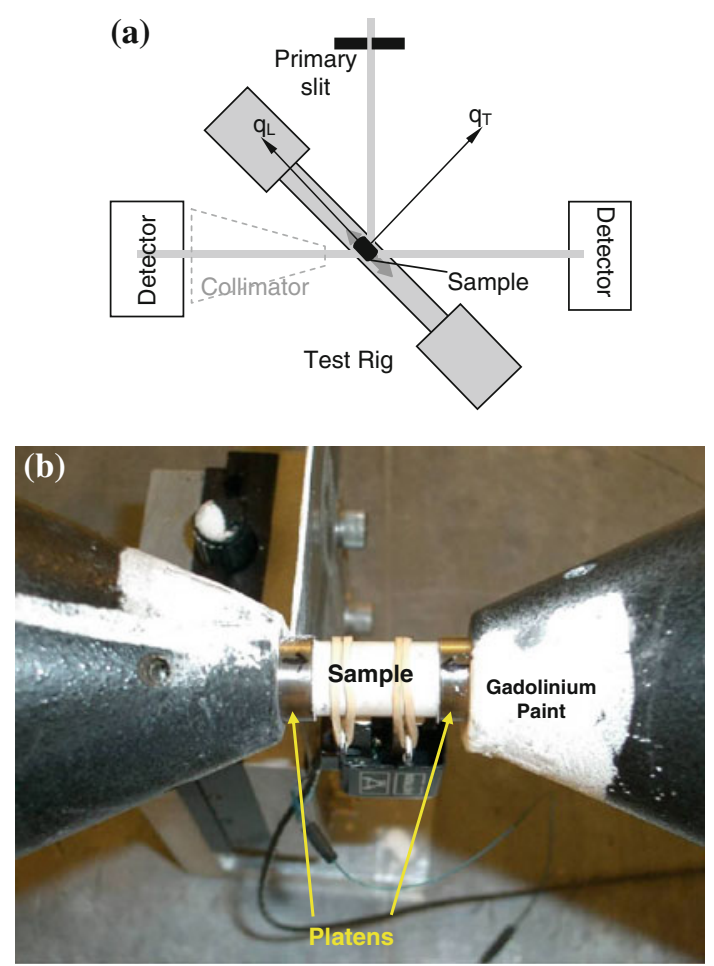

Fig. 6 a Sketch of the diffraction geometry used on SMARTS. The usual position of the radial collimator-not used in the present experiment, is indicated shaded. b Photo of the bulk alumina compression sample mounted on SMARTS at LANSCE

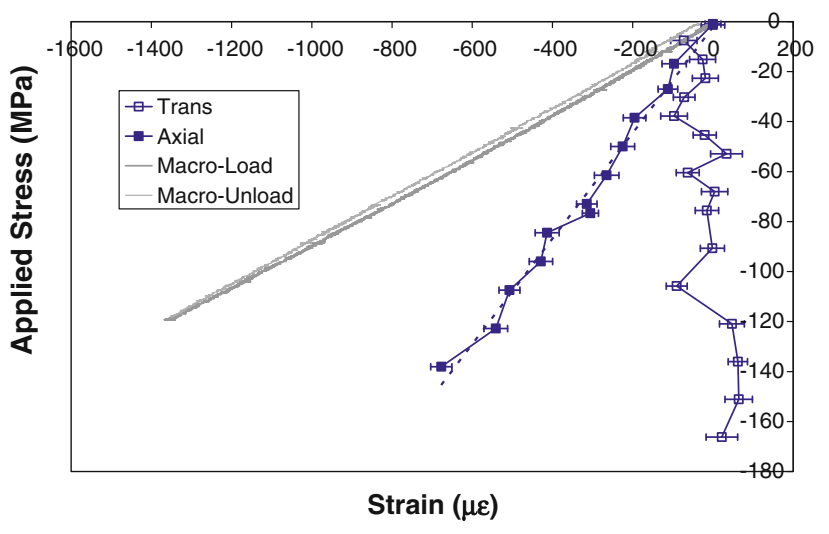

Fig. 7 The evolution of the longitudinal and transverse lattice strain for the hexagonal $116 \mathrm{SiC}$ peak as well as that of the macroscopic strain upon loading and unloading. Error bars are also indicated

transverse direction, because of the smaller amount of deformation.

- The macrostress strain curve is also linear, with a slightly higher slope at low applied stress. The choice of the initial slope would avoid introducing the possible effect of damage on the Young's modulus values [26], but the average slope has been used for the sake of consistency with the ND data treatment. A small but visible hysteresis was indeed noticed for the 
macroscopic curve, which suggests the presence of some mechanical microcracking.

The macro- and microscopic strain of all alumina samples are plotted against the applied stress in Fig. 8. The following comments can be made:

- For all but the $17 \%$ porosity sample, the $a$ - and $c$-axis strains $\left(\varepsilon_{a}\right.$ and $\left.\varepsilon_{c}\right)$ are coincident in the longitudinal direction. On the contrary, for all samples, $\varepsilon_{a}$ and $\varepsilon_{c}$ differ slightly in the transverse direction.

- The microstrain behaviors are almost perfectly linear. Some data scatter can be seen in the $43 \%$ porosity sample, possibly due to the poorer diffraction signal, leading also to larger error bars.

- The macroscopic stress-strain curves are also linear, with the exception of the bulk sample ( $0 \%$ porosity), which seems to have a higher Young's modulus at low applied load. This implies a possible effect of damage on the Young's modulus values [31], analogous to what has been found on SiC. Indeed, the macroscopic curves seem to display some hysteresis, especially at very high porosities.
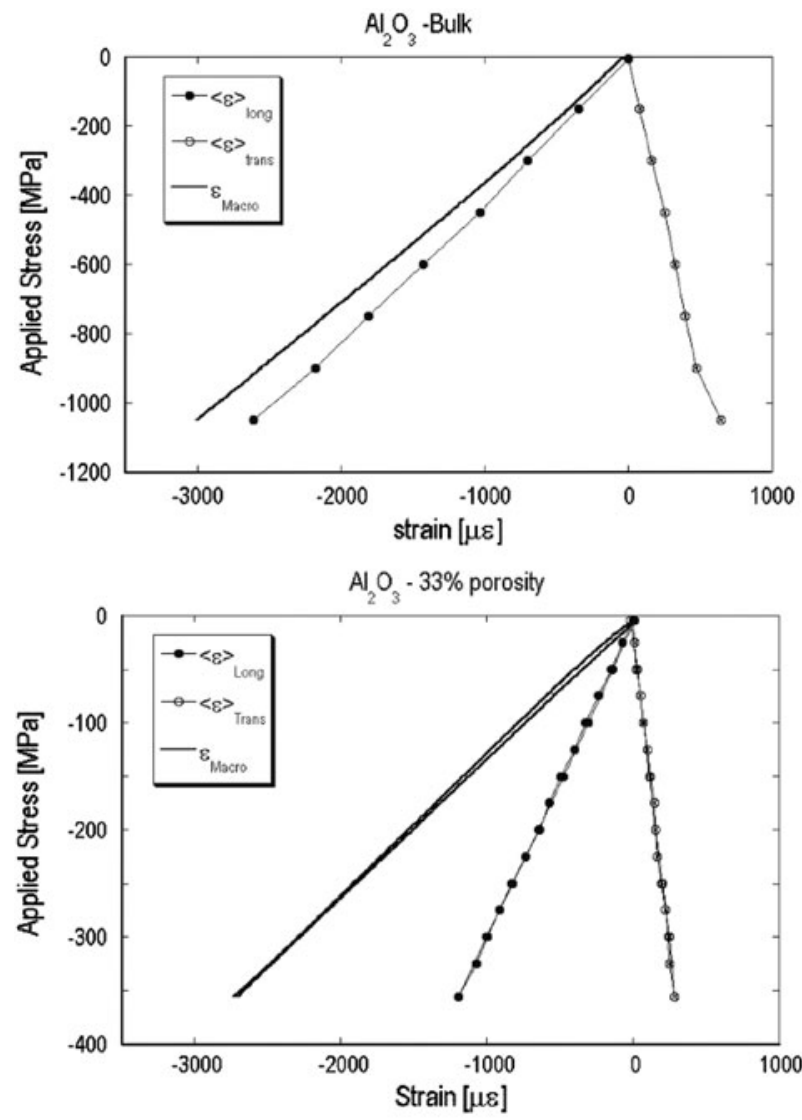

Fig. 8 The macro- and average microscopic strain versus the applied stress for all alumina samples. The strain scale is the same, but the stress range is different for the sake of clarity. The macroscopic
- The (macroscopic) Young's modulus of the dense material $(E=356 \mathrm{GPa})$ is lower than the values of $400 \mathrm{GPa}$ quoted in literature [4, 34]. However, note that the initial slope of the macroscopic stress-strain curve is $395 \mathrm{GPa}$. This confirms that some damage is occurring in the dense alumina sample during the test.

- All microscopic strains show negligible hysteresis.

- The slope difference between the macro- and the microresponse increases as porosity increases.

As mentioned earlier, another important parameter can be extracted from the peak fitting procedure: the integral peak width. This gives the measure of the intra-granular strain and of the grain strain distribution. For steady-state reactor measurements, this is just one fitting parameters of the Gaussian fit function we used. In the case of pulsed sources, a model is required to fit all the integral peak widths at the same time, as shown below.

The longitudinal and transverse peak width dependence from the applied stress for the $\mathrm{SiC}$ is shown in Fig. 9. Interestingly, at high applied stress the integral width in the longitudinal direction increases with the applied load,
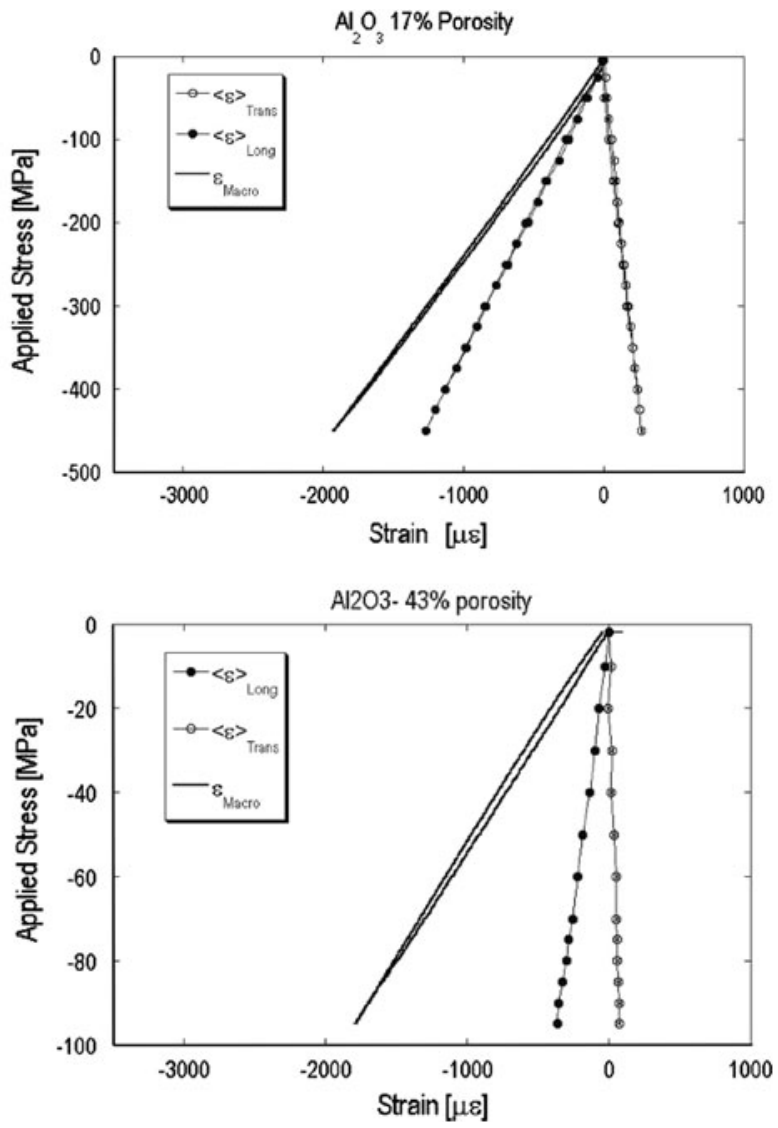

Young's modulus has been evaluated from the average slope. Error bars are contained in the symbols 


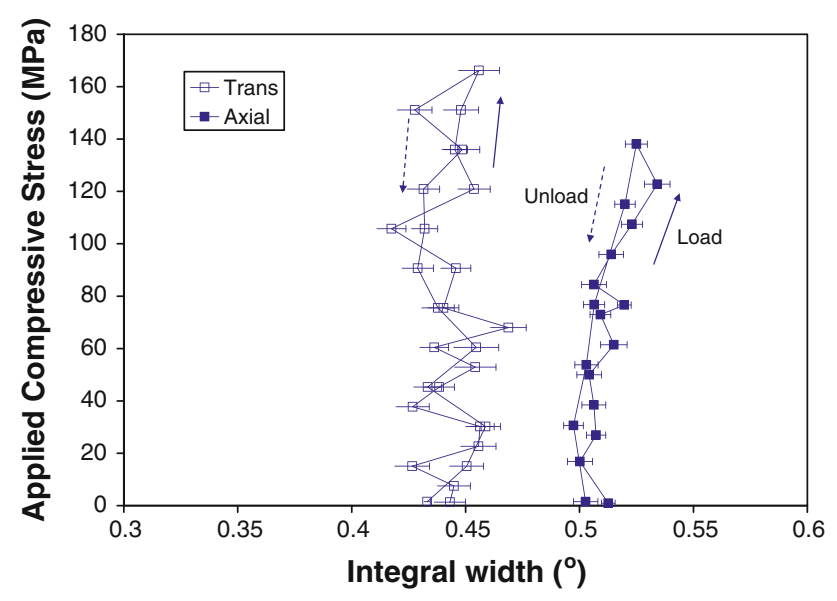

Fig. 9 The evolution of the longitudinal and transverse integral peak width for the hexagonal SiC-116 peak upon loading and unloading. Error bars are also indicated. The increase of the peak width indicates a broadening of microstrain distribution

while it is nearly constant in the transverse direction. This indicates that the strain in the grains always has a larger distribution at high applied stresses, in the longitudinal direction. This is to be expected, since some grains surrounded by pores will basically be unloaded, while others will suffer from stress concentration at sharp edges and will undergo very high stresses. The behavior is even more visible upon unloading, whereby a neat decrease of the longitudinal integral width occurs down to $60 \mathrm{MPa}$ applied stress.

For the alumina, the situation is very similar, with some peculiarities. In the fitting program GSAS [32], the peak width $w$ is modeled as a function of $d$-spacing as

$w^{2}=s_{0}^{2}+s_{1}^{2} d^{2}+s_{2}^{2} d^{4}$

therefore, using three fitting parameters $s_{i}, i=0,2$.

The Gaussian width parameter $s_{1}$ of Eq. 8 was taken to represent the peak integral width, since it is linked to the sample microstrains. Other width parameters, $s_{2}$, linked to microstructural features (crystallite size), or $s_{0}$, linked to instrumental characteristics, were assumed not to vary during the refinement. The behaviors of the peak width of all alumina samples are given in Fig. 10, normalized to the initial value, since the transverse and longitudinal values are slightly different in absolute magnitude.

Interestingly, the integral width in the longitudinal direction increases with the applied stress for all samples, again indicating a larger strain distribution at high applied stresses. This increase is more prominent for high porosities, and has the same explanation as in the case of $\mathrm{SiC}$.

On the contrary, the transverse integral width decreases for the bulk alumina sample, indicating that Poisson's effect induces a narrower strain distribution inside and among the grains. This phenomenon has two main origins: (a) the

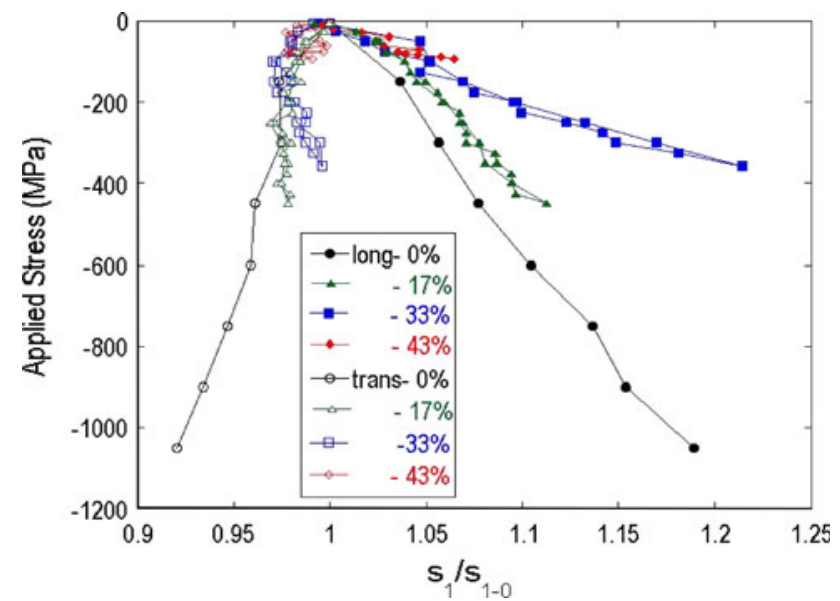

Fig. 10 The normalized integral width parameter $s_{1}$ versus the applied stress for all alumina samples. Both transverse and longitudinal data are reported

residual microstrain in the transverse direction changes in the opposite way to the longitudinal direction; (b) the possible sample texture causes grains to 'see' a different stress field and therefore behaves differently in the two directions. At high porosities, the integral width begins decreasing with the applied load but then stabilizes or increases above a certain applied stress, analogous to what has been observed for SiC. Eventually, for high porosity and high applied load, the increase of the stress distribution width is similar to that in the axial and transverse directions.

\section{Discussion}

Elastic constants

As mentioned above, a suitable value for the comparison between theory and experiment could be the diffraction modulus (also called the diffraction elastic constant) obtained from Eqs. 5 and 6. In fact, the differential form of the longitudinal diffraction modulus for the lattice plane $h k l$ is given by

$E_{h k l, \text { porous }} \equiv \frac{d \sigma_{\text {Macro }}}{d\left(\varepsilon_{h k l, \text { long }}\right)}=E_{h k l, \text { dense }} \cdot(1-p)$

whereby $\varepsilon_{h k l, \text { long }}$ is the measured microstrain and all quantities are evaluated along the longitudinal sample direction (suffix long). The porous modulus is the one we measure, while the dense value may be unknown: Eq. 9 suggests a suitable way to evaluate the dense material elastic constants or the solid phase properties in the porous medium, since the diffraction modulus is not dependent upon the pore morphology.

For the SiC sample ( $p=0.38)$, the dense Young's modulus $E_{\mathrm{d}}$ can be evaluated using the particular 116 lattice 
plane, taking into account that the elastic anisotropy is low for silicon carbide. Indeed, literature data of the $\mathrm{SiC}$ stiffness matrix [35, 36], listed in Table 5, show that the anisotropy factor $\left(E_{111}-E_{116}\right) / E_{116} \sim-11 \%$ as evaluated using a Kröner model [16], is within the experimental error.

We can therefore calculate the macroscopic Young's modulus of the dense material from the measured diffraction Young's modulus of the porous material (221 GPa): $E_{\mathrm{d}}=E_{\mathrm{diff}(116)} /(1-p)=356 \mathrm{GPa}$.

The obtained $E_{\mathrm{d}}$ value falls well into the literature data range (350-375 GPa) for reaction-sintered silicon carbide ceramics http://www.memsnet.org/material/siliconcarbidesic/.

In the case of alumina, the detection of the whole diffraction pattern allows calculating the average microstrain via the unit cell parameters $a$ and $c$ [37]

$\left\langle\varepsilon_{\text {micro }}\right\rangle=\frac{2 \cdot\left\langle\varepsilon_{a}\right\rangle+\left\langle\varepsilon_{c}\right\rangle}{3}$

so that the average diffraction Young's modulus will then be defined as

$\left\langle E_{\text {diff,long }}\right\rangle \equiv \frac{d \sigma_{\text {Macro }}}{d\left\langle\varepsilon_{\text {micro,long }}\right\rangle}=E_{\text {micro,long }} \cdot(1-p)$

The macro- and microscopic Young's moduli for all alumina samples are displayed in Fig. 11 as a function of the dense material fraction $(1-p)$. Interestingly enough, microscopic data show good agreement with the theoretical predictions: the diffraction Young's modulus seems to follow the rule-of-mixtures as a function of porosity.

We also observe that FEM predictions of the microscopic Poisson's ratio agree well with the experimental ND data. This is shown in Fig. 12, where the two FEM models above (OSP and overlapping spherical particles) are compared with the average microscopic Poisson's ratio, defined as

$\langle v\rangle=\frac{\left\langle\varepsilon_{\text {micro,trans }}\right\rangle}{\left\langle\varepsilon_{\text {micro,long }}\right\rangle}=\frac{2\left\langle\varepsilon_{a}^{T}\right\rangle+\left\langle\varepsilon_{c}^{\mathrm{T}}\right\rangle}{2\left\langle\varepsilon_{a}^{\mathrm{L}}\right\rangle+\left\langle\varepsilon_{c}^{\mathrm{L}}\right\rangle}=\frac{\frac{2}{E_{a, \text { diff }}^{\mathrm{L}}}+\frac{1}{E_{c, \text { diff }}^{1}}}{\frac{1}{E_{a, \text { diff }}^{\mathrm{L}}}+\frac{1}{E_{c, \text { diff }}^{\mathrm{L}}}}$

whereby $E_{a, \text { diff }}^{\mathrm{L}}$ is the measured diffraction Young's modulus in the longitudinal direction and the other symbols are consequently defined for the $a$ and $c$-axes in the longitudinal and transverse directions. This value is calculated using the

Table 5 6H-SiC stiffness matrix $C_{i j}(\mathrm{GPa})[35]$

\begin{tabular}{lrrrrrr}
\hline Indices $i, j$ & \multicolumn{1}{c}{1} & \multicolumn{1}{c}{3} & 4 & 5 & 6 \\
\hline 1 & 501 & 111 & 52 & 0 & 0 & 0 \\
2 & 111 & 501 & 52 & 0 & 0 & 0 \\
3 & 52 & 52 & 553 & 0 & 0 & 0 \\
4 & 0 & 0 & 0 & 163 & 0 & 0 \\
5 & 0 & 0 & 0 & 0 & 163 & 0 \\
6 & 0 & 0 & 0 & 0 & 0 & 195 \\
\hline
\end{tabular}

average microscopic strains or taking the ratio of the diffraction Young's moduli in the transverse and longitudinal directions, as defined in Eq. 11. The average of 0.231 is in very good agreement with both macroscopic and polycrystalline microscopic literature values $[4,34,35,38-40]$.

The solid elastic moduli and Poisson ratios obtained from the data in Figs. 7 and 8 are compared in Tables 7 and 8 with the calculated values of the dense material, derived using literature data for the $\mathrm{SiC}$ and $\mathrm{Al}_{2} \mathrm{O}_{3}$ stiffness matrices (Tables 5, 6) http://www.memsnet.org/material/ siliconcarbidesic/. Again, a Kröner's model [16], as

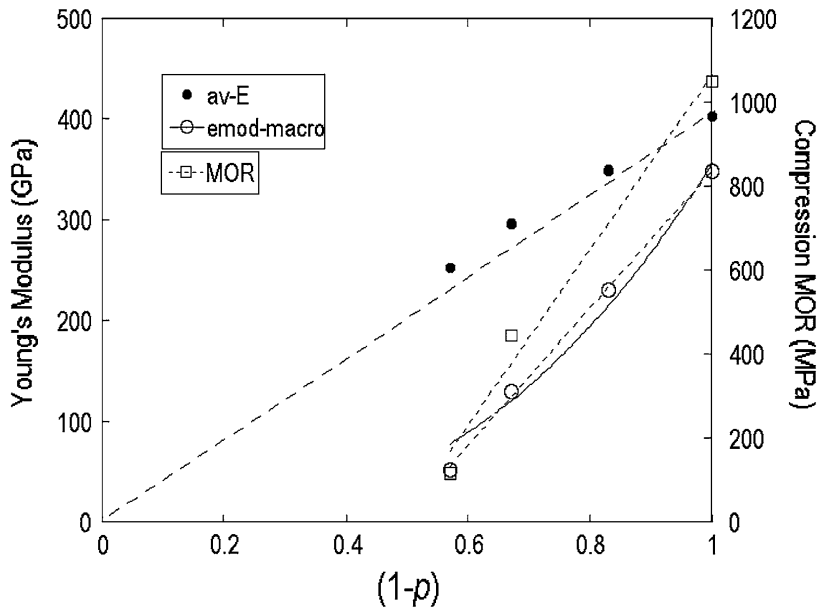

Fig. 11 The macroscopic Young's modulus (open circles), the diffraction average microscopic modulus (full circles), and the compressive strength (MOR, open squares) for $\mathrm{Al}_{2} \mathrm{O}_{3}$ reported as a function of the solid fraction $(1-p)$. The straight dashed line represents the rule-of-mixtures. The solid line is a polynomial fit to the macroscopic Young's modulus values. The dotted lines are linear fits for the MOR and diffraction modulus, getting the same percolation level of $p=0.49$. The error bars for the macroscopic slopes are contained in the symbols

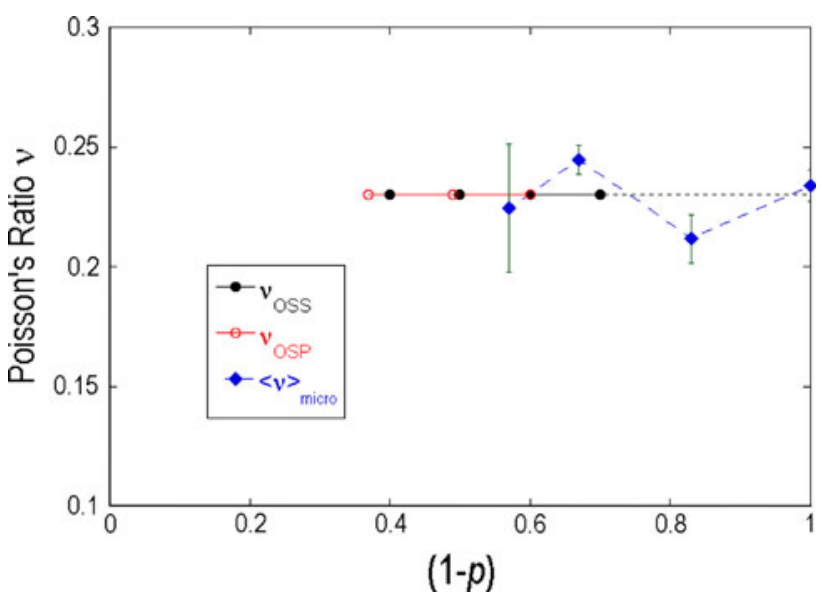

Fig. 12 The microscopic Poisson's ratio, as measured by diffraction and calculated by FEM. $\langle v\rangle$ is the $a$ and $c$-axis weighted average (see text) 
Table $6 \alpha-\mathrm{Al}_{2} \mathrm{O}_{3}$ stiffness matrix $C_{i j}(\mathrm{GPa})$ [38]

\begin{tabular}{lrrrrrc}
\hline Indices $i, j$ & \multicolumn{1}{l}{1} & \multicolumn{1}{l}{3} & \multicolumn{1}{l}{4} & 5 & 6 \\
\hline 1 & 495 & 160 & 115 & 0 & 0 & 0 \\
2 & 160 & 495 & 115 & 0 & 0 & 0 \\
3 & 115 & 115 & 497 & 0 & 0 & 0 \\
4 & 0 & 0 & 0 & 146 & 0 & 0 \\
5 & 0 & 0 & 0 & 0 & 146 & 0 \\
6 & 0 & 0 & 0 & 0 & 0 & 167.5 \\
\hline
\end{tabular}

Table 7 Experimental results for the macroscopic and microscopic Young's moduli, as well as for the Poisson's ratio

\begin{tabular}{llllll}
\hline \multicolumn{3}{l}{ Alumina } & & & $\mathrm{SiC}$ \\
\hline Porosity $\%$ & $43 \%$ & $33 \%$ & $17 \%$ & $0 \%$ & $38 \%$ \\
$E_{\text {diff,long }}(\mathrm{GPa})$ & 252 & 296 & 346 & 403 & 221 \\
$E_{\text {diff,rrans }}(\mathrm{GPa})$ & -1120 & -1212 & -1634 & -1725 & -1842 \\
$E_{\mathrm{M}}(\mathrm{GPa})$ & 50 & 132 & 233 & 352 & 91 \\
$\left\langle-\varepsilon_{\text {micro,trans }}\right\rangle /\left\langle\varepsilon_{\text {micro,long }}\right\rangle$ & 0.22 & 0.24 & 0.21 & 0.23 & 0.12 \\
\hline
\end{tabular}

implemented by the program XEC [39] was used to calculate the polycrystalline averages.

Taking the average derivative $\mathrm{d} \sigma_{\mathrm{M}} / \mathrm{d}\left\langle\varepsilon_{\text {micro }}\right\rangle$ over the whole lattice strain versus applied stress curve, and the average slope for the macroscopic stress-strain curves, we obtained the results given in Table 7. In addition, Table 8 shows that the agreement between model, experiments and literature values is very good.

One further comment can be made: the slight nonlinearity of the macroscopic stress-strain curve in Figs. 7 and 8 implies a degradation of the sample stiffness at high load. This could be related to local damage of the structure at the points of high stress. Indeed, we have seen that the peak width in the longitudinal direction shows an increase at high applied load (Figs. 9, 10). At macroscopic level (see Eq. 1) the nonlinearity should be interpreted as an increase of the pore morphology factor $m$ due to microdamage.

Pore morphology factor

The pore morphology factor $m$ could be derived from the micro/macrostrain ratio obtained by the ND experiments reported above, in two different ways: (a) from the ratio of macroscopic porous to dense material elastic constants, and (b) by macroscopic stress-strain tests, with the input of literature values for dense material properties:

a. Using the ratio between micro- and macroscopic strain, i.e., using Eq. 6, we get

$$
m_{\text {micro }}=1+\frac{\ln \left(\frac{\left\langle\varepsilon_{\text {micro,long }}\right\rangle}{\varepsilon_{\text {Macro }}}\right)}{\ln (1-p)}
$$

The ratio of the micro- to macrostrain was shown to be independent of the solid phase elastic constants, and therefore of literature values, which may not correspond to the processing route of the investigated materials.

b. Using the classical macroscopic approach, i.e., taking for the Young's modulus the measured macroscopic $\left(E_{\text {Macro }}\right)$ and the literature value (e.g., $E_{\mathrm{d}}=400 \mathrm{GPa}$ for dense alumina) we have from Eq. 1.

$$
m_{\text {Macro }}=\frac{\ln \left(\frac{E_{\mathrm{M}}}{E_{\mathrm{d}}}\right)}{\ln (1-p)}
$$

$m_{\text {micro }}$ and $m_{\text {Macro }}$ are tabulated in Table 9. The two sets of values agree very well for $\mathrm{SiC}$ and reasonably well for alumina: in the latter case, for high porosity we have only $8 \%$ difference, while at low porosity we have a mismatch in excess of $30 \%$. This possibly implies that the solid domain elastic properties in highly porous materials differ from the literature value for dense alumina.

All the $m$ values are intermediate between the OSP and the OSS structures, if we compare them with FEM results; this is also suggested by the $\mathrm{SiC}$ micrograph in Fig. 4. This consistency is further evidence for the model validity.

For alumina, some further comments must be made: the plot of the macroscopic and microscopic Young's moduli versus $(1-p)$ (Fig. 11) allows one to fit an average exponent $m$ for the macroscopic structure as $m=2.71 \pm$ 0.35 . This indicates that, expectedly, alumina possesses

Table 9 Derived values for the pore morphology factor

\begin{tabular}{lllll}
\hline Porosity $\%$ & Alumina & & $\mathrm{SiC}$ \\
\cline { 2 - 4 } & $43 \%$ & $33 \%$ & $17 \%$ & $38 \%$ \\
\hline$m_{\text {Macro }}$ & 3.47 & 2.46 & 2.21 & 2.85 \\
$m_{\text {micro }}$ & 3.77 & 3.01 & 3.49 & 2.83 \\
\hline
\end{tabular}

Table 8 Calculated values of the dense material elastic coefficients (Young's modulus and Poisson's ratio) for alumina

\begin{tabular}{llllllll}
\hline & Porosity & & & & Average & & $\begin{array}{c}\text { Literature [38] } \\
\text { + Kröner [16] }\end{array}$ \\
\cline { 2 - 5 } & 0.43 & 0.33 & 0.17 & 0 & & $\begin{array}{l}\text { Literature } \\
\text { macro [4] }\end{array}$ \\
\hline$E_{\mathrm{d}}(\mathrm{GPa})$ & 415 & 440 & 419 & 405 & $420 \pm 21$ & 420 & 400 \\
$\langle v\rangle$ & 0.198 & 0.247 & 0.231 & 0.247 & $0.231 \pm 0.023$ & 0.231 & 0.23 \\
\hline
\end{tabular}


neither spherical $(m=2)$ nor star-like pores, $m=4$ (i.e., its porosity is generated by overlapping spherical particles). However, from Fig. 11 we notice that the macroscopic Young's modulus of the $43 \%$ porosity alumina lies off the fitting curve more than the other points do. This can be explained by the fact that the sintering parameters (temperature and time), required to obtain lower porosity materials from initial powders, played an important role in defining the pore morphology factor.

It is not the goal of this paper to go into the details of the sintering process (see $[41,42]$ as examples). While the curvature of the pores is constant for this kind of ceramics in the intermediate stage of sintering and theoretically tends to zero [43] due to surface energy minimization, the pore morphology factor changes. This can be formalized if we take the different $m_{\text {Macro }}$ values listed in Table 9 and compare them with the average $m$ quoted above. The

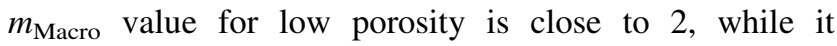
increases at higher $p$. This would introduce a dependency of the pore shape factor $m$ on the porosity itself. However, materials with very different porosities may have similar pore morphology factors or vice versa. Therefore, the connection between $p$ and $m$ is fictitious, since both truly depend on the processing route. In fact, as Green reports in [12], a linear dependence of the macroscopic Young's modulus as a function of porosity could also be attached to our data, getting a percolation value of $p_{0}=0.49$. This is represented in Fig. 11 (dashed curve) and implies that strictly speaking the pore morphology factor used in this work is not directly linked to the curvature of the pores. This also can explain why in the literature all the models proposed (see the introduction: exponential, rational, linear, power) equally fit the experimental data. We further notice that whichever model is used, the compression MOR seems to follow the elastic coefficient law as a function of porosity [44] (i.e., it has for instance the same percolation level in the linear model).

It is fundamental to control the sintering conditions in such a way to have comparable pore morphology factor, if we want to assess the universal dependence from porosity of the elastic properties of a material in the classical manner used by Gibson and Ashby [21]. This is also one of the most important findings of the investigations of Wang [40]. In fact, the method proposed in this work (see Eq. 13) is a way to evaluate the pore morphology factor deconvoluting the pore shape from the porosity value. Tomography work would obviously helps to assess the influence of the sintering parameters on the pore morphology, but this is again beyond the scope of this work.

One of the main limitations of the analytical model is its scalar nature, which contrasts with the tensorial character of the stress state in a porous material. The bridge between the two worlds is laid down by the FEM calculations.
Although based on a few different model microstructures, they yield insights into the general features of the stress state in the material. These general features are independent of the detailed microstructure of the solid, since the latter is even more complicated.

In conclusion, we can state that the agreement between literature, calculated and measured data allows a reliable use of the latter to extract the properties of the dense material, which would be inaccessible to solely macroscopic techniques.

\section{Summary and conclusions}

In this work, analytical modeling of the macro- to microstrain and stress conversion has been carried out for porous ceramics. This approach has been based on stress balance in elastically isotropic media. It has been shown that the macro-stress to average micro-stress ratio depends linearly on porosity and does not depend on pore morphology factor $m$, while the ratio of macro-strain to average microstrain does. The factor $m$ and the Young's modulus of the dense material, both can be extracted from uniaxial compression testing, if the porosity is known. Thus, the model suggests a way to evaluate the elastic constants of the dense material using experiments on the corresponding porous material.

FEM calculations have allowed cross-checking the analytical calculation for different model microstructures. The agreement between the two theoretical approaches proved to be astounding. In addition, the FEM approach allowed calculating shear strains arising during uniaxial testing and visualizing the behavior of the pore structure under load.

ND in situ compression testing carried out on porous honeycomb $\mathrm{SiC}$ bars and on compact porous alumina cylinders has fully validated the model and the FEM calculations. Experimental results show that the macroscopic and microscopic strain responses to external stress are not equal: they reflect the peculiarities of the whole body deformation (including pores) and the grain response, respectively. For alumina, we have shown that, knowing the macroscopic Young's modulus of the dense material, values of the pore morphology factor $m$ could be extracted in two different ways: as a common value to all samples, following a power law, or as individual values. The mismatch between the two approaches gives a cross-check of the assumption that pores have the same morphology factor for a material with different porosities. Moreover, it has been shown that using the microscopic strain response, a novel method to estimate the pore morphology factor could be proposed, which is independent of the literature's Young's modulus values. 
FEM confirmed the experimental finding that the microscopic Poisson's ratio does not vary as a function of porosity and pore morphology factor, and it is always equal to the macroscopic value for the dense material. The average measured value for alumina was $0.23 \pm 0.02$, which agrees very well with the calculated and literature value of 0.23 .

The approach has proven to be self-consistent. Through the use of ND, the link found here between average microscopic and macroscopic strains and stresses in porous media opens new opportunities for the estimation of macroscopic and microscopic crystalline mechanical properties of the correspondent dense material.

Acknowledgements Thomas Glasson and Cedric LeGoff (Corning SAS, CETC, Avon, France), Angela Graefe, Andy Schermerhorn, James E. Webb and Lisa Noni (Corning Inc, Painted Post, NY, USA.), Irina Pozdnyakova (CNRS, Orleans, France), and Darren J Hughes (ILL, Grenoble, France), Donald W. Brown and Thomas A. Sisneros (MST-8, LANL, Los Alamos, NM, USA) are kindly acknowledged. This work has benefited from beam time from the Institut LaueLangevin (ILL), Grenoble, France, as well as the use of the Lujan Neutron Scattering Center at LANSCE, which is funded by the Office of Basic Energy Sciences (DOE). Los Alamos National Laboratory is operated by Los Alamos National Security LLC under DOE Contract DE AC52 06NA25396.

\section{References}

1. Green DJ, Nader C, Brezny R (1990) In: Handwerker CA, Blendell JE, Gysser WA (eds) Ceramic transactions, vol 7, Sintering of advanced ceramics. American Ceramic Society, Westerville

2. Knudsen FP (1962) J Am Ceram Soc 45:94

3. Spriggs RM (1961) J Am Ceram Soc 44:628

4. Pabst W, Gregorová E (2006) In: Caruta BM (ed) Ceramics and composite materials. Nova Science Publishers, New York, pp 31-100

5. Dean EA, Lopez JA (1983) J Am Ceram Soc 66:366

6. Munro RG (2001) J Am Ceram Soc 84:1190

7. Green DJ (1998) An introduction to the mechanical properties of ceramics. Cambridge University Press, Cambridge, UK, pp 88-94

8. Wang JC (1984) J Mater Sci 19:809. doi:10.1007/BF00540452

9. Sudduth RD (1995) J Mater Sci 30:4451. doi:10.1007/ BF00361531

10. Ramakrishnan N, Arunachalam VS (1990) J Mater Sci 25:3930. doi:10.1007/BF00582462

11. Roberts AP, Garboczi EJ (2000) J Am Ceram Soc 83:3041
12. Kachanov M, Tsukrov I, Shafiro B (1994) Appl Mech Rev 47:151

13. Frishbutter A, Neov D, Scheffzük C, Vrana M, Walther K (2000) J Struct Geol 22:1587

14. Darling TW, TenCate JA, Brown DW, Clausen B, Vogel SC (2004) Geophys Res Lett 31:16604

15. Nikitin AN, Ivankina TI, Soboilev GA, Scheffzük C, Frishbutter A, Walther K (2004) Izvestyia Phys Sol Earth 40:83 (translated from Fizika Zemli (2004) 1:88)

16. Kröner E (1958) Z Phys 151:504

17. Reuss A (1929) Z Angew Math Mech 9:49

18. Berryman JG (1997) Phys Rev Lett 79:1142

19. Gebert JM, Wanner A, Piat R, Guichard M, Rieck S, Paluszynski B, Böhlke T (2008) Mech Adv Mater Struct 15:467

20. Batzle ML, Simmons G, Siegfried RW (1980) J Geophys Res 85:7072

21. Gibson LJ, Ashby MF (1982) Proc R Soc Lond A382:43-59

22. Pabst W, Gregorova E (2007) Ceram Int 33:9

23. Phani K, Niyogi SK (1987) J Mater Sci 22:257. doi:10.1007/ BF01160581

24. Timoshenko S, Woinowsky-Krieger S (1959) Theory of plates and shells. Springer, New York

25. Rossi RC (1968) J Am Ceram Soc 51:433

26. Pozdnyakova I, Bruno G, Efremov AM, Clausen B, Hughes DJ (2009) Adv Eng Mater 11:1023

27. Hauk V (1997) Structural and residual stress analysis by nondestructive methods: evaluation — application—assessment. Elsevier Science, Amsterdam

28. Noyan IC, Cohen JB (1987) Residual stress: measurement by diffraction and interpretation. Springer-Verlag, New York

29. Webster PJ (1991) In: Hutchings MT, Krawitz AD (eds) Measurement of residual and applied stress using neutron diffraction. NATO Series E 216, Kluwer Academics, Dordrecht

30. Hughes DJ, Bruno G, Pirling T, Withers PJ (2006) Neutron News $17: 28$

31. Rietveld HM (1968) J Appl Crystallogr 2:65

32. Larson AC, Von Dreele RB (2004) General structure analysis system (GSAS). LANL Report LAUR 86-748

33. Bourke MAM, Dunand DC, Üstündag E (2002) Appl Phys A 74:S1707

34. Pabst W, Gregorová E (2004) J Mater Sci 39:3501. doi:10.1023/ B:JMSC.0000026961.12735.2a

35. Kamitani K, Grimsditch M, Nipko JC, Loong CK, Okada M, Kimura I (1997) J Appl Phys 82:3152

36. Tolpygo KB (1961) Sov Phys Solid State 2:2367

37. Daymond MR, Bourke MAM, Von Dreele RB (1999) J Appl Phys 85:739

38. Tefft WE (1966) J Res Natl Bur Stand 70A:277

39. Wern H (2000) XEC Program, Ottweiler, D

40. Wang JC (1984) J Mater Sci 19:801. doi:10.1007/BF00540451

41. Chaix JM (2009) Mater Sci Forum 624:1

42. Pampuch R (1979) Ceram Int 5:76

43. Jung Y, Chu KT, Torquato S (2007) J Comput Phys 223:711

44. Knudsen FP (1959) J Am Ceram Soc 42:376 